\title{
Paradoxical buckling behaviour of a thin cylindrical shell under axial compression
}

\author{
E.R. Lancaster ${ }^{1}$, C.R. Calladine*, S.C. Palmer ${ }^{2}$ \\ Department of Engineering, University of Cambridge, Cambridge CB2 IPZ, UK
}

Received 15 May 1998; received in revised form 8 March 1999

\begin{abstract}
The widely accepted theory of buckling of thin cylindrical shells under axial compressive loading emphasises the sensitivity of the buckling load to the presence of initial imperfections. These imperfections are conventionally taken to be minor geometric perturbations of a shell which is initially stress-free. The original aim of the present study was to investigate the effect on the buckling load of imperfections in the form of local initial stress, which are probably more typical of practice than purely geometric ones. Experiments were performed on a vertical "melinex" cylinder of diameter $\sim 0.9 \mathrm{~m}$ and height $\sim 0.7 \mathrm{~m}$, with radius/thickness ratio $\sim 1800$. The upper and lower edges of the cylinder were clamped to end discs by means of circumferential belts - an arrangement that allowed states of self-stress to be introduced to the shell readily by means of local "uplift" at the base. The upper disc was made sufficiently heavy to buckle the shell, and it was supported by a vertical central rod under screw control. Many buckling tests were performed. Surprisingly, the buckling loads were generally at the upper end of the range of fractions of the classical buckling load that have been found in many previous experimental studies. Even when the local uplift at the base caused a local "dimple" to be formed before the shell was loaded, the buckling load was relatively high. A surface-scanning apparatus allowed the geometric form of the shell to be monitored, and the progress of such a dimple to be followed; and it was found that a dimple generally grew in size and migrated in a stable fashion up the shell as the load increased, until a point was reached when unstable buckling occurred. These unexpected and paradoxical features of the behaviour of the experimental shell may be attributed to the particular boundary conditions of the shell, which provide in effect statically determinate support conditions. This study raises some new issues in the field of shell buckling, both for the understanding of buckling phenomena and for the rational design of shells by engineers against buckling. (C) 2000 Elsevier Science Ltd. All rights reserved.
\end{abstract}

Keywords: Cylindrical shell; Buckling; Axial load; Imperfections; Self-stress imperfections; Experiments; Statically determinate boundary conditions

\footnotetext{
* Corresponding author. Tel.: 01223-332725.

E-mail address: crc@eng.cam.ac.uk (C.R. Calladine)

${ }^{1}$ Present address: Group Risk Engineering, Zurich Insurance Company, TRP Unit, Southwood Crescent, Farnborough, GU140NJ, UK.

${ }^{2}$ Present address: Yarm School, Stockton-on-Tees, TS15 9EJ, UK.
} 


\section{Nomenclature}

$\begin{array}{ll}a & \text { radius of shell } \\ E & \text { Young's modulus of material } \\ t & \text { thickness of shell } \\ \theta & \text { circumferential angular coordinate } \\ v & \text { Poisson's ratio of material } \\ \sigma_{\mathrm{b}} & \text { measured buckling stress } \\ \sigma_{\mathrm{c} 1} & \text { classical buckling stress }\end{array}$

\section{Introduction}

Much has been written on the topic of the buckling of thin cylindrical shells subjected to axial compressive loading. Ever since the discovery, almost 60 years ago, that buckling loads measured by experiment generally fell far short of the predictions made by the classical, elastic, small-deflection eigenvalue buckling theory, research workers have studied various aspects of the shell-buckling problem. As von Kármán [1] argued at the time, geometric nonlinearity must play an important part in the phenomenon of thin-shell buckling. And for the past 30 years or so, Koiter's [2] conceptual scheme involving unstable equilibrium paths emerging from a bifurcation point for the initially perfect cylindrical shell, and the presence of small geometrical imperfections having a disproportionately large effect in reducing the maximum load, has become the conventional wisdom for engineers. That scheme, which connects analytically (i) an imperfection sensitivity of the buckling load, (ii) the "scatter" of measured peak-load values and (iii) highly unstable post-buckling, has furnished a useful qualitative picture of shell buckling. On the other hand, extensive studies aimed at the prediction of buckling loads for cylindrical shells on the basis of precisely measured geometric imperfections [3] have had only limited success [4]. It seems reasonable to conclude, therefore, that the current conventional wisdom concerning the buckling of thin shells omits some crucial physical factor from the analytical scheme.

The experimental study to be described in the present paper forms part of an ongoing research project which aims at investigating this putative "missing ingredient" in current thinking about shell buckling. The project explores the idea that, while imperfections in the construction of thin shells may indeed be responsible for the low and scattered practical buckling loads, the kind of stress-free, geometric imperfections that are envisaged in the conventional theory $[2,3]$ are by no means the only kind of practical imperfection in shells as actually built. And indeed it might be argued that these "conventional" imperfections are rather unlikely to be found in practical construction.

An obvious candidate for a significant constructional imperfection is one in which the shell as built is in a state of initial stress. Such initial stress could, in principle, predispose a shell to buckle under a small (or even zero) applied load. In what circumstances, then, can a thin cylindrical shell sustain a state of initial stress, or self-stress? Now it may sometimes be argued that a thin shell is statically determinate when it is idealised as a membrane, since the three independent equilibrium 
equations that are obtained by resolving forces on an arbitrary small element in three perpendicular directions are sufficient to determine, for arbitrary loading, the three independent components of the membrane stress throughout the shell [5]. And a statically determinate structure is, by definition, incapable of sustaining a state of self-stress [6]. But such an argument cannot be sustained in general, even within the membrane hypothesis, because it overlooks the role of displacement boundary conditions in fixing the determinancy or indeterminancy of the statical equilibrium equations: there is no reason to suppose that displacement boundary conditions have less influence with shells than with, say, simple triangulated frameworks, where they can render statically indeterminate an otherwise statically determinate frame.

The following simple example illustrates the point at issue here. An open-topped thin-walled circular cylindrical crude-oil storage tank, made from pre-curved flat sheet and initially stress-free, will distort in a more-or-less stress-free manner if small non-uniform vertical displacements are imposed at its base on account of uneven settlement of the foundation. The shell responds to such changes practically inextensionally, with the generators of the surface remaining straight, and with a radial inward movement at the top in direct proportion to the upward vertical displacement of the corresponding point at the base [7, Section 5.1]. However, if the same pattern of vertical base-displacements is imposed at the bottom of a similar shell that is now fitted with a closed roof, then the situation is very different. The circularity imposed by the roof at the top prevents the inextensional pattern of distortion; and therefore the displacements imposed at the base must cause stresses to be set up within the shell. The key difference between the two situations is that the boundary conditions of the second tank (base held circular and plane; top held circular) make the shell statically indeterminate, and hence capable of sustaining a state of self-stress; while those of the first tank (base held circular and plane; top free) make the shell statically determinate, and hence incapable of sustaining a state of self-stress. It follows immediately from this analysis that, in a tank with its top held circular (but free to distort out of plane) and its bottom held both circular and plane, it is possible for significant stresses to be set up by small, additional, out-of-plane movements of the base.

The main purpose of the experiments to be described in this paper was to determine the effect on the buckling load for such a shell of small, locally applied "uplift" at the base of the shell. All of our experiments were performed on a cylindrical shell of diameter $\sim 0.9 \mathrm{~m}$ and height $\sim 0.7 \mathrm{~m}$, made from a rectangular sheet of "melinex" of thickness $0.25 \mathrm{~mm}$ : radius/thickness $\approx 1800$.

In view of what has been stated above, we expected that "uplift" would introduce locally a region of high compressive stress, that would in turn predispose the shell to buckle in an unstable fashion under lower axial load than in the absence of uplift. However, the actual behaviour of the shell turned out to be quite different from such expectations in various respects. First, the initial uplift at the base tended to produce a stable, local, inward-directed "dimple" in the shell at a low axial load - or even at zero axial load, depending on the degree of uplift. In one sense a buckle had indeed appeared at low or zero axial load. However, when the axial load was increased, this dimple tended to grow larger both in superficial extent and in depth, and to move gradually up the shell, towards mid-height. Throughout this process the behaviour was clearly stable. Then, eventually, the shell would suddenly buckle comprehensively at a particular value of axial load, leaving much of its surface covered by a more-or-less regular pattern of post-buckling dimples, as shown in Fig. 1. We clearly need to be careful about our terminology. We have already described as a "stable dimple" something that might otherwise be called a "buckle". But for the remainder of this paper we shall 


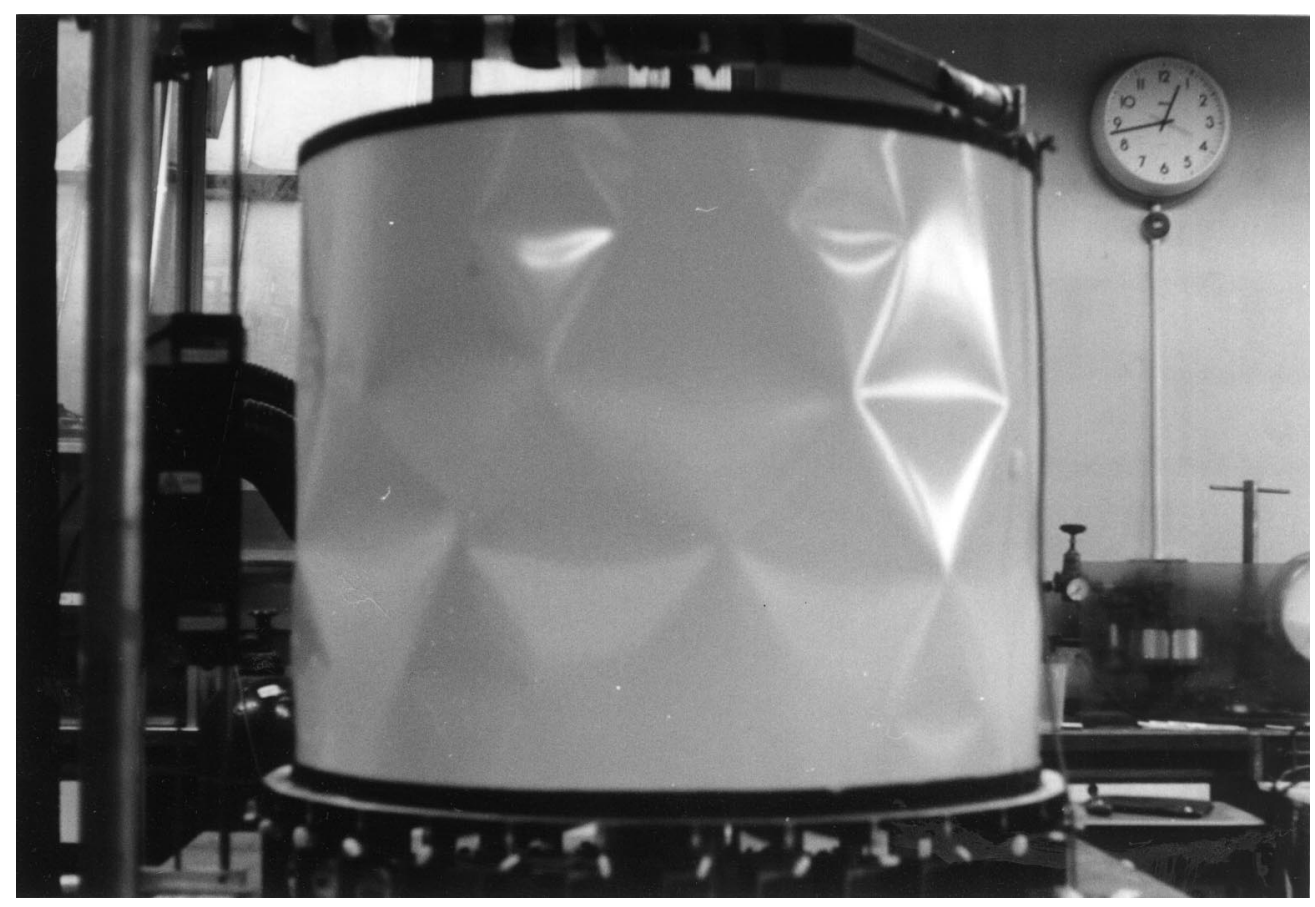

Fig. 1. Photograph of shell in post-buckled state, in which approximately half of the circumference is covered by a more-or-less regular pattern of "dimples".

use the term "buckling" only to indicate the point where the applied axial load reaches a maximum, and then falls into a post-buckling state in which the shell is covered with many dimples.

A second surprising observation was that the buckling loads recorded in many tests conducted without any local uplift at the base were almost as high, in relation to the predicted classical value, as any that have been recorded in the literature for shells having similar radius/thickness values. And indeed there was relatively little "scatter" in the observed buckling loads. In general, the consequence of introducing local uplift at the base in many other tests was to depress the average buckling load somewhat; but again there was relatively little "scatter" in the buckling loads. Why then did our shell perform so well, in spite of its deliberately introduced imperfections?

A third surprising feature of our experiments was that the buckling load of the shell was so high, in spite of a rather awkward longitudinal seam (see below), which clearly constituted a major geometrical imperfection, in the sense described by Koiter.

In view of these various experimental observations, all of which run counter to Koiter's conceptual scheme as described above, our research project developed in a direction that was different from the original aim. Thus, the unexpected absence of significant scatter in the observed buckling loads, contrary to the conclusions of many previous studies, raised a question requiring an explanation. Eventually we realised that a key to the situation lay in the somewhat unusual boundary conditions at the upper and lower ends with which we had set up the shell, primarily as a matter of experimental convenience: they will be described below. We came to believe that an 
explanation of the unexpected observations must lie in a proper understanding of some subtle features of our chosen boundary arrangements.

The layout of the paper is as follows. First we describe the experimental apparatus and the shell, including the boundary arrangements and the means of measuring axial load and the current geometrical conformation of the shell. Then we describe the measured initial geometry of the shell as it was set up. Section 3 gives first a statistical treatment of buckling loads reached in a total of 30 tests made with the shell set up in a "stress-free" condition, and then a description of a similar number of tests made after uplift-imperfections had been deliberately introduced. Section 4 discusses our results in relation to other work.

\section{Apparatus and measurement arrangements}

\subsection{General arrangement}

The main features of the experimental apparatus are shown schematically in Fig. 2. The base of the shell is supported on a "table-top" consisting of a thin steel plate attached to 32 equally spaced radial bars whose levels may be adjusted individually by means of thumbscrews. This part of the apparatus had been built by Kamyab [8] for an experimental study of the way in which a small-scale melinex model of an open-topped crude-oil storage tank distorts when the base settles in an uneven fashion; and in particular it enabled him to impose arbitrary patterns of non-uniform

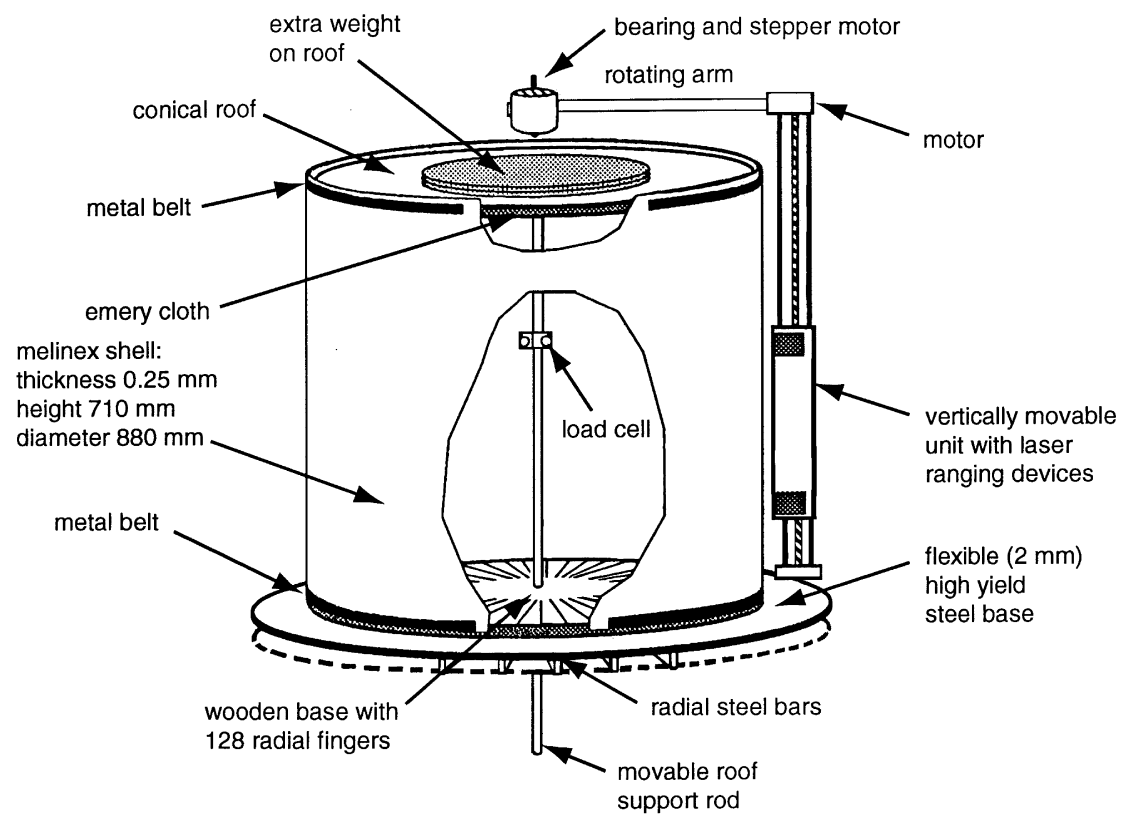

Fig. 2. Schematic view of apparatus and scanning device, with parts of the shell cut away in order to show various features. At the bottom of Fig. 1 may be seen parts of the screw devices for adjusting the level of the individual radial bars, which have not been shown here (note that the scanning device cannot be seen in Fig. 1). 
settlement onto his model shell at 32 "stations". Our intention was to use Kamyab's heightadjusting arrangement to induce self-stress imperfections in the shell, as described above; but in practice (see below) we had to adapt the apparatus somewhat so that it could impose local uplift at the base of the shell.

The shell itself was made from a single sheet of melinex, cut accurately into a rectangular shape. The two short edges were abutted together, and the vertical seam was made by gluing over the edges a cover-strip, $12 \mathrm{~mm}$ wide, of the same material; Evo-stick impact adhesive was used.

The upper end-closure "roof" of the shell consisted of a very shallow steel cone, $1.5 \mathrm{~mm}$ thick, welded inside an outer rim of external diameter $880 \mathrm{~mm}$, with rectangular cross-section of height 25 $\mathrm{mm}$ and thickness $5 \mathrm{~mm}$. The overall weight of the upper disc, having been increased by the symmetrical addition of masses, was more than sufficient to buckle the shell. The axial force applied to the shell was controlled by supporting the centre of the upper disc on a long central rod that could be raised and lowered at its lower end by means of a hand-controlled screw, and which incorporated a load-cell for measuring the axial force. At any time, therefore, the axial load applied to the shell was equal to the total weight of the upper disc less the force measured in the rod. In the course of a typical test the lower end of the rod was gradually lowered by means of the screw so that the compressive force in the rod was steadily reduced, and the load on the shell correspondingly increased. When the shell reached its peak load-carrying capacity, it buckled; and the shell's surface suddenly became filled with post-buckling dimples as described above (see Fig. 1). At this stage the presence of the rod limited the vertical drop of the upper disc, and thus prevented a catastrophic failure of the shell. In practice, at this stage the upper disc tilted on its central support, so that about half of the shell surface was covered with buckles while the other half was practically unbuckled. Although the single shell specimen was subjected to buckling on many different occasions, there was no evidence that repeated buckling produced any permanent damage or weakening; thus the buckles appeared to be always within the elastic range of the melinex material.

\subsection{Connecting the shell to its upper and lower discs}

As we have already indicated, boundary conditions at the top and bottom edges of the cylindrical shell were of critical importance to our investigations. The easiest way of explaining the procedures that were used for fixing these edges is to imagine a smaller-scale paper model of the cylinder made by taking half of an A4 sheet of paper cut lengthways down the middle, rolling it into a cylindrical shape and gluing the short edges together with a small overlap. This paper cylinder is floppy, and is able to undergo substantial inextensional distortion. Suppose that two circular balsa-wood discs, 2 or $3 \mathrm{~mm}$ thick, and of negligible weight, are now cut so that they just fit inside the ends of the small paper cylinder. A lot will depend on the precise size of these discs; but they can be made to fit inside the cylinder easily, yet with enough friction just to hold the upper disc in place when the assembly is stood up vertically. It is reasonable to suppose that in this condition the paper of the cylinder will be stress-free; and indeed that it would remain stress-free if the discs could be somehow connected permanently to the cylinder in a way that would produce no local disturbance. A possible way of joining the disc and the cylinder would be to coat the disc (made slightly smaller, perhaps) with a slow-setting glue, so that as the glue hardens the stress-free condition persists. (It would be important, of course - just as in making the longitudinal seam of the cylinder - to use a kind of glue that does not cause the paper to swell or shrink, or otherwise distort.) 


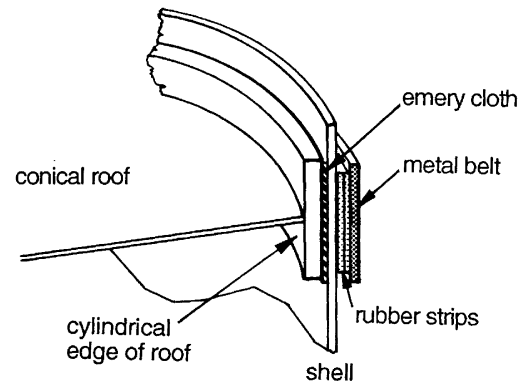

(a)

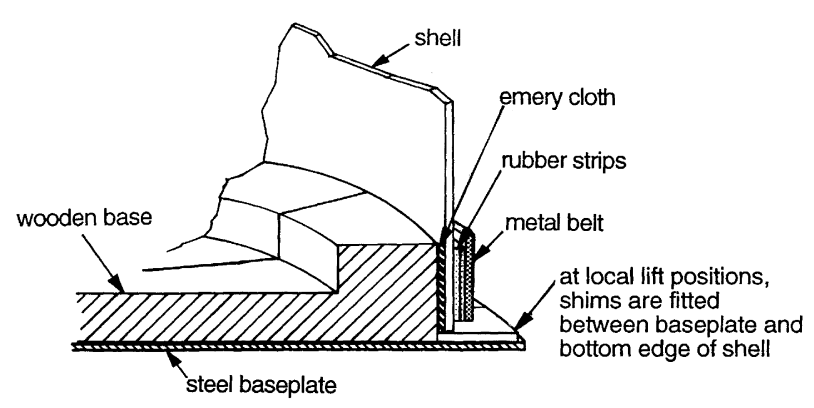

(b)

Fig. 3. Local cut-away schematic views showing details of the clamping arrangements at (a) the top and (b) the base of the shell. See text for explanation.

In relation to the problem of fixing the heavy roof-disc to the $0.25 \mathrm{~mm}$ thick melinex cylinder in the actual test rig, the use of glue was considered initially. However, since it would clearly be desirable in a project of this kind to be able to detach and re-fix the roof at will during the course of investigations, it was decided to use a more reversible fixing arrangement. The basic idea was to clamp the shell to the $25 \mathrm{~mm}$ deep cylindrical edge of the upper disc by means of a thin circumferential aluminium belt equipped with a simple turnbuckle, and separated from the melinex sheet itself by two compressible rubber strips, together about $3 \mathrm{~mm}$ thick: see Fig. 3(a). In terms of the small-scale paper cylinder, this would be equivalent to securing the end-discs by placing a taut elastic band around the cylinder, in the plane of each disc. The fixing would obviously not be as positive as a glued joint; but if the tension in the band were sufficiently high, the grip might be sufficiently firm for buckling experiments to be performed satisfactorily. In order to improve the grip between the melinex shell and the end-discs in our experiments, the cylindrical edges of the roof disc and the lower disc (see below) were both covered with emery cloth, grade 400, attached with glue.

The support conditions at the base of the shell were more difficult to arrange than those at the top, on account of our intention to impose small vertical displacements there after fixing. After some trials of several different schemes, the following arrangements were made (see Fig. 3(b)). First, a built-up circular plywood disc, $25 \mathrm{~mm}$ thick at its outer edge, was cut to exactly the same overall diameter as the upper roof disc. Then a set of radial slits, $0.3 \mathrm{~m}$ long, were cut into the disc from the outside, so that the outer part of the disc was transformed into 128 radial "fingers" - i.e. 4 for each circumferential "station" of the flexible steel base plate described above. These separate fingers were secured to the base plate with double-sided adhesive tape; and in addition every fourth finger was held to the plate by screws. The initial plan was that the melinex sheet would be firmly attached to the ends of the fingers, and that, as Kamyab's baseplate-adjusting screws were turned, the fingers, and hence the shell, would move vertically by prescribed small amounts, as required. It turned out, however, that the grip between the melinex sheet and the end surfaces of the fingers covered with emery cloth, as a result of pressure from the circumferential securing belt similar to the one at the top, was not sufficiently positive to avoid slip; and so an additional arrangement was 
incorporated to ensure that the vertical lift imposed on the base was positively transferred to the shell. As before, the cylinder was clamped to the wooden blocks by means of a circumferential belt with a turnbuckle, but now leaving a gap of about $2 \mathrm{~mm}$ between the bottom of the shell and the base plate. Then, at a location where the base of the shell was to be raised, specially cut shims were inserted between the shell and the base. So, when the corresponding base-plate-adjusting screws were turned, a small portion of the circumference of the shell was raised directly. This arrangement is shown in the sketch of Fig. 3(b); and it was found to work satisfactorily.

\subsection{Setting up the shell for a test}

The setting-up procedure for the shell was rather involved, and it was gradually developed by a process of trial and error. First, the shell was slipped over the wooden base and clamped to it lightly by means of the rubber-lined aluminium belt (Fig. 3(b)). Care was taken to ensure that the lower edge of the shell was not in direct contact with the steel base plate, so as to allow some shims to be inserted later on. During this fitting process, the roof was supported clear of the shell on the central rod, which could be raised and lowered manually by means of the screw arrangement already described. The roof was then lowered gently, and carefully slipped inside the top edge of the shell. When the roof was at its proper height it was clamped to the shell by the upper aluminium belt (Fig. 3(a)). After the shell had been set up in this way, either belt could be released temporarily (but only one at a time) and the shell's position adjusted relative to the corresponding disc. The alternating loosening and re-clamping of the two belts allowed for release of any locked-in stresses, and so provided an initial stress-free condition in the shell before each test.

\subsection{Surface position-measuring system}

An important aspect of the experimental apparatus, that we have so far mentioned only in passing, was a device for systematically scanning the radial position of the shell, at any stage during a test. The scanning arrangement is shown schematically in Fig. 2: it was adapted, with substantial modification, from Kamyab's apparatus.

Above the centre of the top of the shell is located a vertical bearing which supports a rotating radial arm. The bearing is supported on a simple frame built from $50 \mathrm{~mm}$ diameter steel scaffolding tubing and proprietary junction units, and firmly attached to the laboratory floor. The scaffolding framework stands clear of the table supporting the base plate and the shell, and is independent from it.

The rotating arm is driven by a stepper-motor controlled by a computer. From the end of the arm projects downwards a long vertical member consisting of two rods that form a "track" for a movable unit holding two laser ranging devices. This unit is driven vertically from the top by a motor that turns a long vertical screwed rod. The basic scanning plan was for the arm to rotate steadily while the laser-carrying unit climbed steadily from the bottom, so that the lasers could take a series of readings of the radial position of the shell on slowly climbing, spiral paths. Initially, a single laser was used to scan the full height of the shell. With this arrangement, however, a complete scan of the shell took almost one hour. In order to speed up the process the carriage was modified so that it supported two laser ranging units, separated by a fixed distance a little less than half of the height of the shell. In this way each laser scanned half of the surface of the shell. 
Initially, the signals from the lasers were communicated to the recording computer by means of slip-rings. But such signals turned out to be "noisy"; and so a scheme of direct wiring was set up instead. This involved a helically coiled "telephone cord" connection lying on the roof of the shell, which could twist up without distress through the necessary total number of revolutions as the scan proceeded. After each scan was complete, this cord was disconnected and untwisted in preparation for the next scan. A straight vertical cable on the outside of the shell, close to the seam, brought the signals to the base of the shell, and thence to the computer. The final scheme used for scanning the shell surface had the radial arm turning steadily under the action of the stepper motor, with readings of the laser rangers being taken at predetermined angular positions. The fact that the laser was moving over the surface as readings were taken was found not to affect the accuracy of the readings.

The motor controlling the vertical motion of the laser-carriage was a simple d.c. machine, and its voltage was carefully adjusted in order to achieve the required uniform speed of climb. A simple scheme for controlling the motors and the lasers was set up, and it worked well. The laser devices were light and compact and could measure the surface at a range of $100 \pm 40 \mathrm{~mm}$, with a resolution of $50 \mu \mathrm{m}$ and at a response interval of $20 \mathrm{~ms}$. Lasers with visible beams were used to ensure safety and to locate properly the starting positions on the shell.

The scanning apparatus was programmed to perform 18 circumferential circuits, in each of which 128 equally spaced radial measurements were taken. The long spiral traverse was preceded by, and followed by, a circular scan. The measurements were thus taken on a grid with vertical and circumferential spacings of 21 and $22 \mathrm{~mm}$, respectively. It was necessary to "match" the first circuit of reading of the upper laser with the last circuit of the lower laser: but this caused no difficulty.

Since the bearing of the scanner was not necessarily located precisely over the centre of the shell, a simple procedure was devised for locating the true centre of the data points at top and bottom by use of the fundamental Fourier coefficients of the data. In general the raw data on radial position were improved - i.e. were less noisy — when a three-point running average was used along the spiral.

\subsection{Plotting schemes}

The plotting scheme that we have found most useful is the cartesian plot on the "unrolled" shell, as exemplified by Fig. 4. Here the radius of the shell is plotted vertically on the page, the scale being marked in $\mathrm{mm}$; the average radius is $440 \mathrm{~mm}$. The circumferential and axial scales are also marked in $\mathrm{mm}$. Note that the axial coordinate has been "stretched" by a factor of about two; and so the elementary "squares" of the measured grid $(21 \times 22 \mathrm{~mm})$ appear to be approximately twice as high as they are wide. The scale of radial displacement is exaggerated by a factor of about 70 in comparison with the circumferential scale.

Fig. 4(a) and (b) show two views of the same data set, from inside and outside the cylinder, respectively: note the labelling of the radial axes. The data set corresponds to a shell with a substantial dimple in the near, right-hand corner of the plot. The dimple, which is seen to be roughly as wide as it is high on the shell's surface, presents itself here as an elongated "hump" in the interior view (a) on account of the different scales used in the plotting scheme. This "hump" is flanked by two "hollows" in Fig. 4(a), but these now appear as separate "humps" in (b); and in that view the dimple itself is a deep "hollow", hidden behind the nearer "hump". It is clear from these 

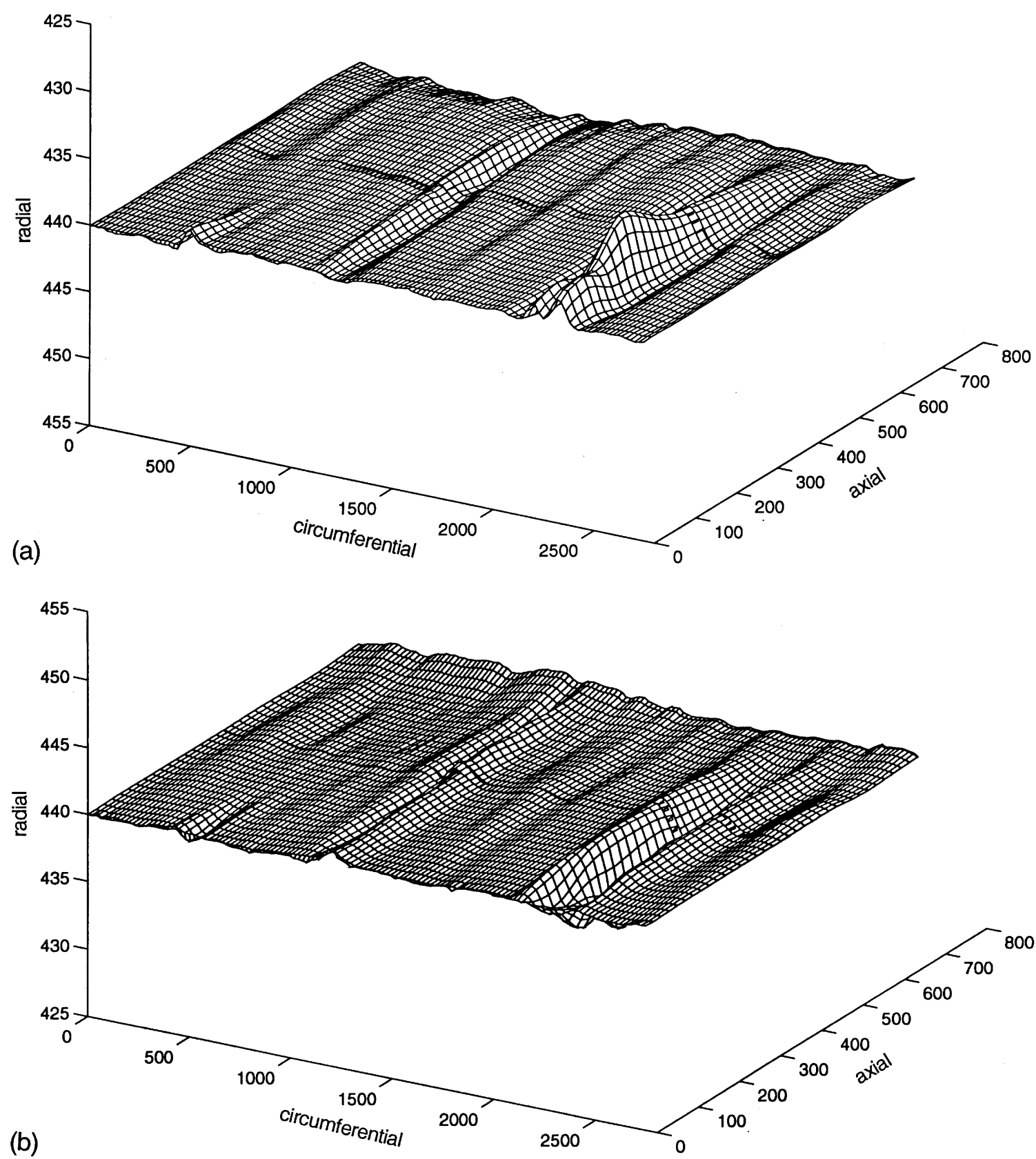

Fig. 4. Cartesian plots showing the conformation of the shell at a particular stage during a test. The radial, axial and circumferential co-ordinate axes are all graduated in $\mathrm{mm}$, but with different scales. In (a) the surface is viewed from the inside and in (b) from the outside of the shell. There is a localised dimple in the lower right-hand corner. The conspicuous feature just to the left of centre is the axial seam. 
two views that some experience is needed for the interpretation of cartesian plots of this kind; and that inside- and outside-views provide complementary information.

Several other features are visible in Fig. 4; and most of them are also present in the shell when it has been set up initially in a stress-free condition. The most conspicuous feature is the full-height vertical seam of the shell. Viewed from the inside we see an inward-directed displacement of the centre of the seam, while the view from outside shows two compensating ridges of outwards displacement on either side. The amplitude of the seam-ripple is approximately $0.5-0.7 \mathrm{~mm}$, i.e. two or three shell thicknesses. The butt joint with its cover strip evidently pulls the shell inwards locally (a "sinkage"), evidently on account of a "curling" effect in the cover strip and adhesive over the course of time.

Another visible feature of Fig. 4 is a set of well-defined regular ripples, that usually have an amplitude of about $0.3 \mathrm{~mm}$, or about one shell thickness. In practice, after the shell had been set up - i.e. clamped to the upper and lower end discs - it was used for several tests, and hence it was scanned several times before being set up again. In general, the pattern of ripples did not change much until the shell was set up again by adjustment of the end fixings. The ripples were not generally in phase with the seam, so they cannot be regarded as a consequence of that feature. Simple inspection of the profiles and/or Fourier analysis reveals a prominent mode with circumferential wave-number $n \approx 10$, together with some contributions at $n \approx 14$. A straightforward calculation [7, problem 8.1] indicates that a simply supported cylindrical shell of the present dimensions has its most flexible long-wave mode at $n \approx 12.5$. The most likely cause of the rippling is the small amount of circumferential shortening — of the order of $0.1 \mathrm{~mm}$ altogether — induced at the ends of the shell by the tightening of the clamping-belts; and indeed in some scans there are signs of additional local rippling effects near the turnbuckles.

Another scheme of plotting data is shown in Fig. 5(a). Here also the cylinder has been "unrolled", with axial height plotted vertically and circumference horizontally. The axial scale has been "stretched" by a factor of about three with respect to the circumferential scale. The curves correspond to contours of radius - at a spacing of about $4 \mathrm{~mm}$ - and they show the shell in a many-dimpled post-buckled form: see Fig. 1. Each elliptical patch (which would be nearly circular if the vertical and circumferential scales had been equal) corresponds to an inward-directed dimple, while the intervening elongated regions correspond to outward-directed vertical "creases". It is clear that in the main the dimples are aligned vertically; and indeed that there is a rough regularity among the dimples that cover the buckled portion of the shell. A cartesian plot (cf. Fig. 4) of the same post-buckled configuration, as viewed from the inside of the shell, is shown in Fig. 5(b). The many "peaks" correspond to the inward-directed dimples.

An obvious feature of the actual post-buckled shape (Fig. 1) is that each of the many dimples has a clearly defined straight, horizontal "crease" across its centre. This crease is straight because the surface has been inverted locally in an almost inextensional way into a portion of a horizontal cylinder. This well-defined geometrical feature is not at all obvious in either the cartesian or the contour plot. However, once it is appreciated that every such dimple contains a straight, horizontal crease, this can be recognised in the "standard", practically parabolic, circumferential profiles of the "humps" on the cartesian plot (Figs. 5(b) and 4(a)) that correspond to inward-directed dimples.

A third kind of plot is shown in Fig. 6. This is a "true-view" plot of a relatively small portion of the circumference of the shell. It shows a well-defined single dimple, as in Fig. 4, which has a generally diamond-shaped perimeter. In Fig. 6 the vertical scale on the page has been exaggerated 

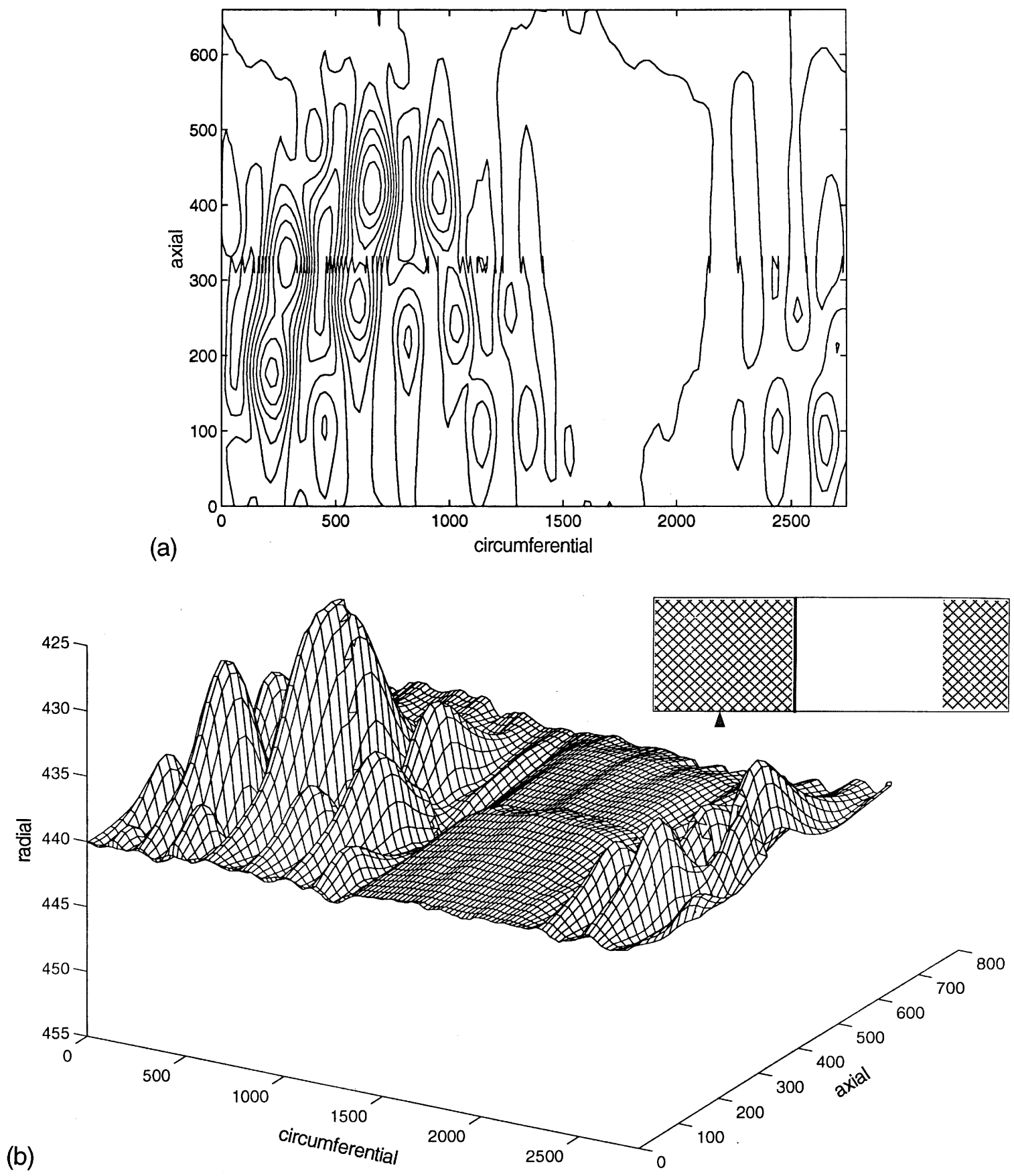

Fig. 5. (a) Contour plot of the post-buckled surface of the shell, as in Fig. 1. (b) Corresponding cartesian plot of a similar post-buckled state. The elliptical patches in (a) and the "humps" in (b) correspond to the diamond-shaped dimples of Fig. 1. The inset diagram indicates schematically the major features of deformation. 


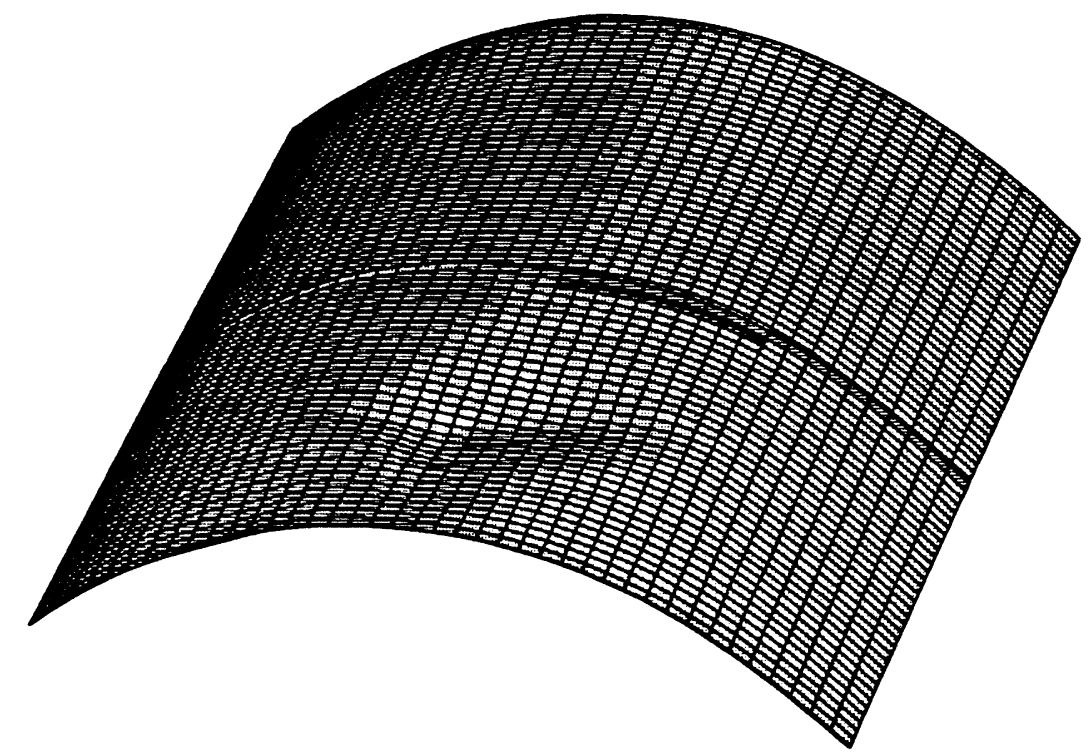

Fig. 6. A "true-view" plot of a portion of the shell containing an isolated dimple, as in Fig. 4. The vertical (i.e. practically radial) scale of the picture has been exaggerated to emphasise the indentation. The grid is half the size of that shown in Fig. 4, by interpolation.

- in this case by a factor of about 2. Note that the circumferential "crease" that crosses the dimple is preserved as a straight feature in this view, however much the view is "stretched". But also note that the Cartesian plot of Fig. 4(a) indicates that the single dimple has a long "tail" that extends practically to the top of the shell - a feature which is not at all obvious in the plot of Fig. 6.

It is clear from all of this that each of the three different schemes of plotting has both advantages and disadvantages. For the remainder of the paper we shall use the cartesian plot exclusively.

\section{Experimental observations}

\subsection{General}

Several different kinds of test were performed on the shell. Most tests began with the shell set up in a nominally stress-free state by adjustment of the upper and lower clamping belts as described in Section 2. Some tests were done without use of the scanning equipment, while in other tests scans were made at several different stages.

Altogether some 64 tests were performed. In the simplest tests - 30 in number - the shell was set up in a nominally stress-free state; then the load was steadily increased until the shell buckled; and the peak load was recorded. The mean buckling load recorded in this way was $436 \mathrm{~N}$, with a standard deviation of $23 \mathrm{~N}$. These values correspond to 0.36 and 0.02 of the classical buckling load, respectively, which we have taken as $1200 \mathrm{~N}$ (see the appendix). Fig. 7 shows a histogram of these observed buckling loads. 


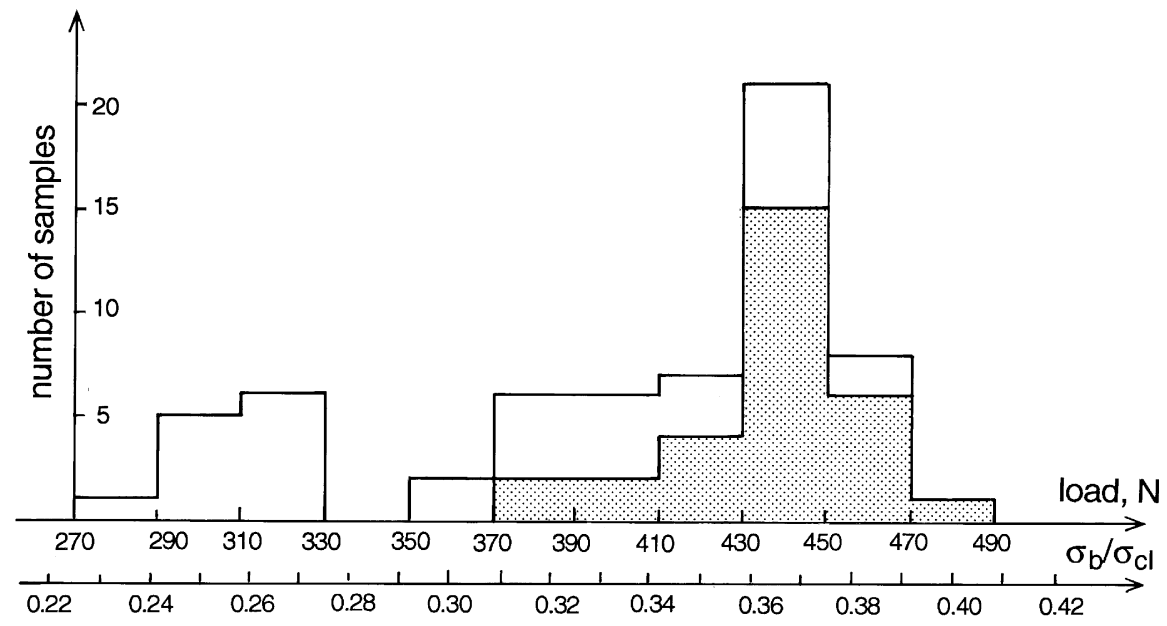

Fig. 7. Combined histogram of buckling loads measured in 64 tests. The horizontal scale represents the buckling load expressed both in Newtons and as a fraction the classical buckling load of $1200 \mathrm{~N}$ for the shell. The shaded part corresponds to 30 tests in which no imperfection was introduced, while the unshaded part represents 34 tests in which imperfections were introduced. Means and standard deviations for the two sets of data are given in the text.

In order to compare these data with buckling loads observed by many workers in previous studies, we have shown in Fig. 8 the range of our experimental observations on an existing plot [9] of a collection of buckling loads shown as a fraction of the classical value, against the value of radius/thickness. The solid bar corresponds to the range of our test results on nominally stress-free specimens. Our data range from 0.31 to 0.41 of the classical value, whereas the collected data from past tests for similar values of radius/thickness range from about 0.1 to 0.4 of the classical value. Thus all of our data lie in the upper third of the spectrum of previously collected data.

We performed altogether 34 buckling tests after various kinds of imperfection had been introduced - mainly by lifting the shell locally at one or more points around the base. Taking all of these tests together, we obtain a spread of buckling loads from 0.23 to 0.39 of the classical value, with a mean of 0.31 and a standard deviation of 0.05 . The range of results has also been shown in Fig. 8 by an open bar; and the buckling loads evidently lie in the upper half of the "collected data", in spite of the deliberately introduced imperfections.

These buckling-load results are clearly paradoxical in relation both to the collected data from previous tests and also in relation to Koiter's conceptual scheme of buckling. Thus, for example, several versions of Koiter's theory predict a buckling load of around 0.4 of the classical value when there is a geometrical imperfection of radial amplitude only 0.2 times the thickness of the shell (see, e.g. Refs. [7, Fig. 15.11; 10]); whereas, our shell has a clear imperfection in geometrical form at the axial seam of amplitude about two or three thicknesses.

But perhaps the most striking and surprising feature of our data is the excellent performance of our shell even after significant imperfections have been deliberately introduced. Thus, in some cases the deliberate uplift introduced at the base produced a dimple in the unloaded shell that had an inwards radial displacement of about $4 \mathrm{~mm}$ or 16 thicknesses; yet the shell typically supported 


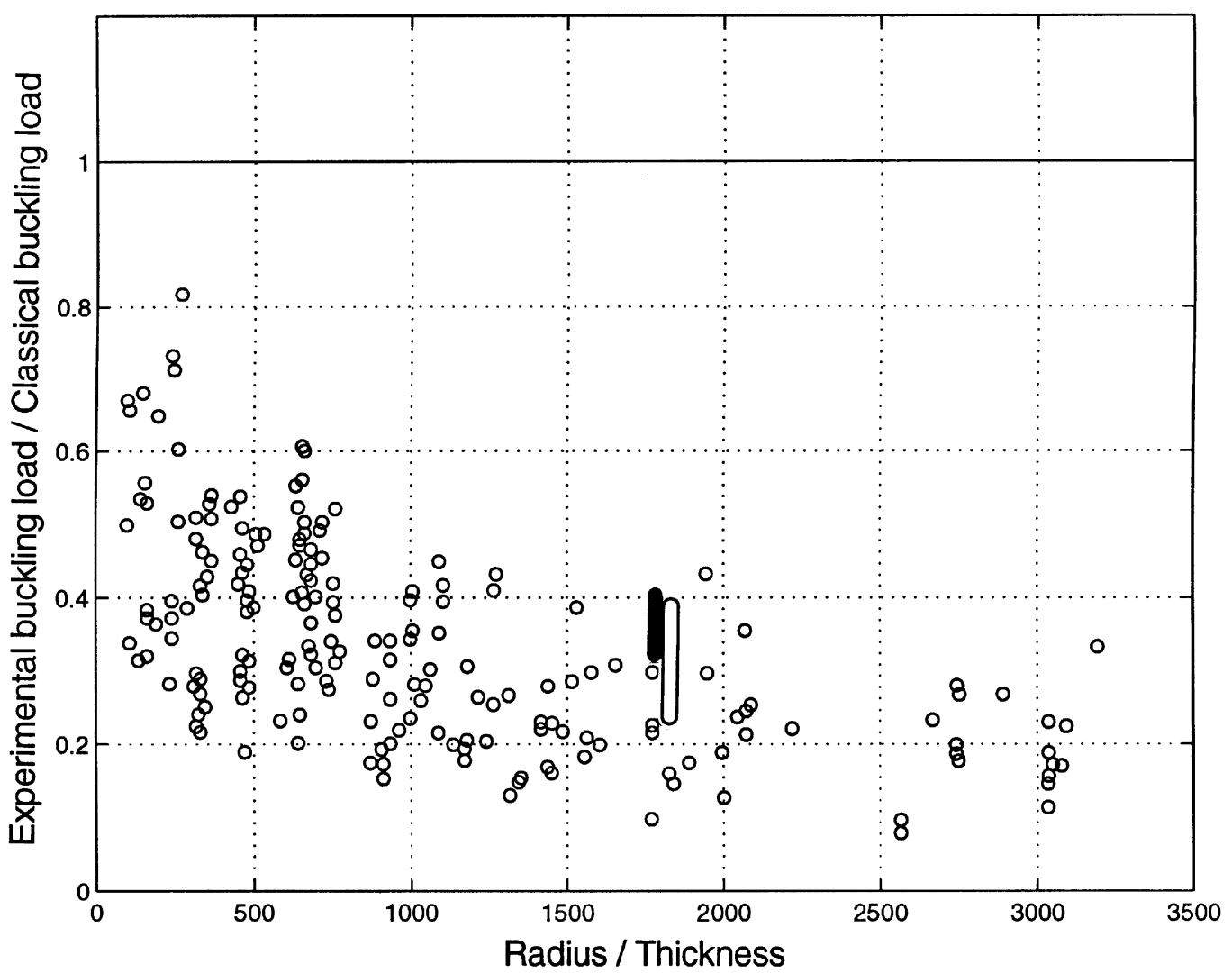

Fig. 8. Buckling-load data from many experiments on axially compressed cylindrical shells, plotted as a fraction of the classical buckling stress against the radius/thickness ratio $R / t ; E$ is Young's modulus of the material: from Ref. [9, p. 186]. The ranges of our data for shells with deliberate imperfections (open bar) and without (solid bar) (see Fig. 7) are also shown.

a load of 0.29 of the classical value before it buckled - a value approximately equal to the mean of all our tests on the shell with deliberate imperfections (see above).

\subsection{A particular case}

In order to highlight another paradoxical aspect of our results, we now describe in detail a particular, but typical, test in which we imposed a local uplift on the shell at its base, and which provided a clearly visible dimple before the shell was loaded. We made a scan of the shell in this condition, and then we made further scans as the axial load applied to the shell was increased by increments of $70 \mathrm{~N}$.

The various scans are displayed in Fig. 9(a)-(f). They show clearly that at zero load there is a dimple above the point of uplift; and that when the load is increased the dimple simultaneously enlarges and migrates up the shell. Under the largest axial load of $350 \mathrm{~N}$ the dimple has a depth of about $8 \mathrm{~mm}$ and a circumferential width of about $100 \mathrm{~mm}$. In this particular test the shell was left 

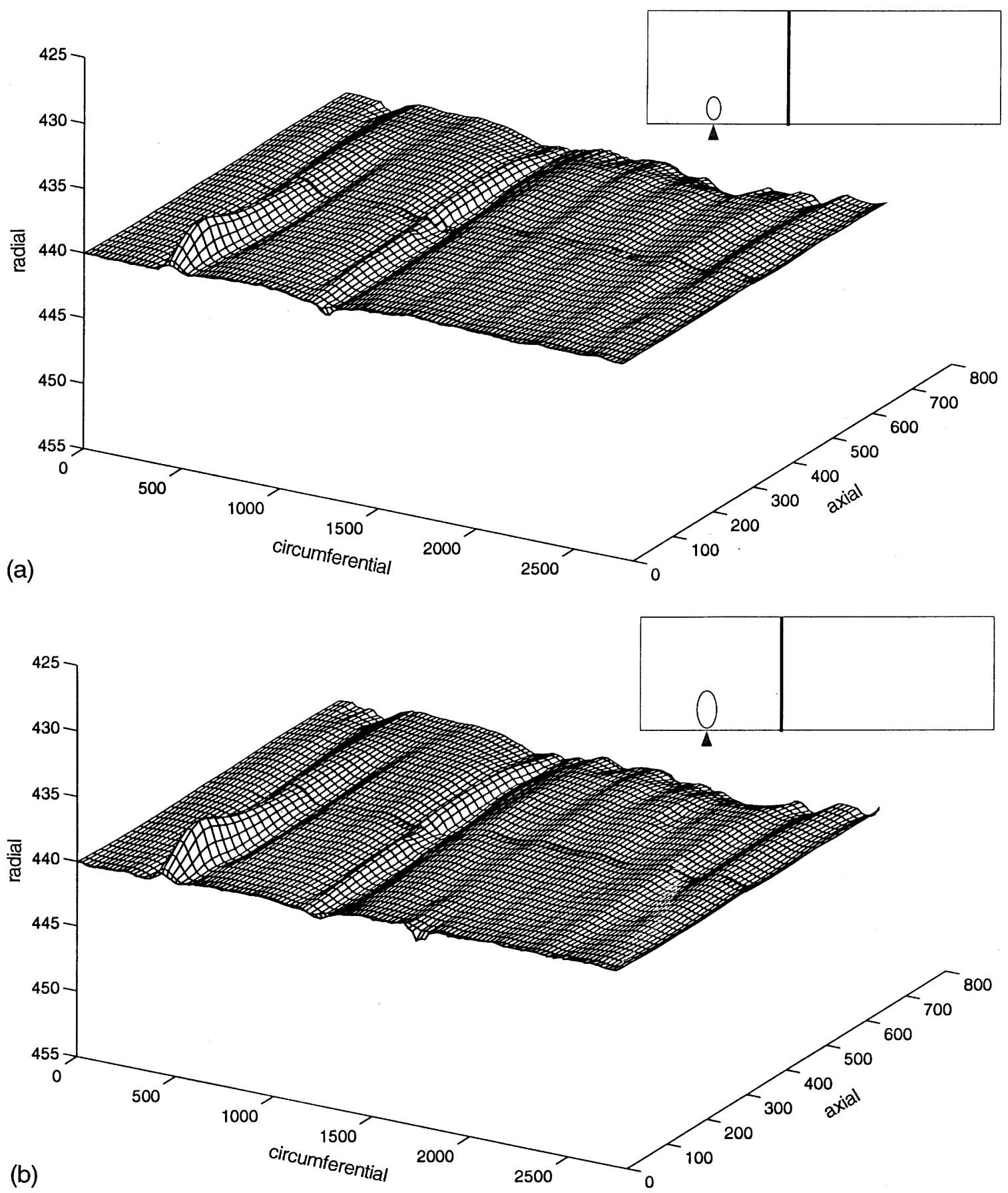

Fig. 9. Cartesian plots of a test in which an uplift of $0.2 \mathrm{~mm}$ was imposed on the shell under zero load (a), and then the load was increased to $350 \mathrm{~N}$ in equal steps of $70 \mathrm{~N}$ (b-f). The inset diagrams indicate schematically the growth of the dimple, but without showing the horizontal crease across its centre (cf. Figs. 1 and 6). 

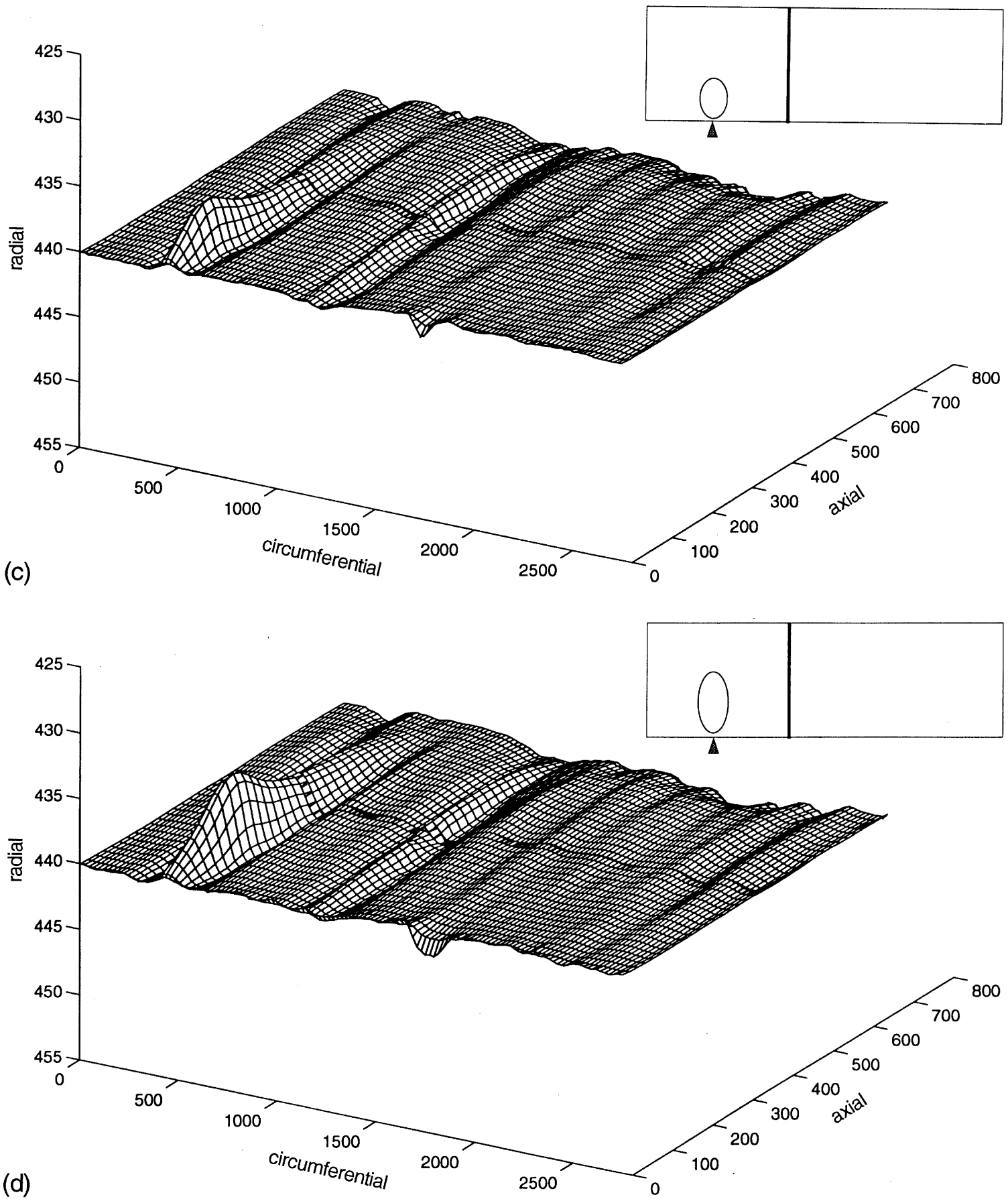

Fig. 9. continued. 

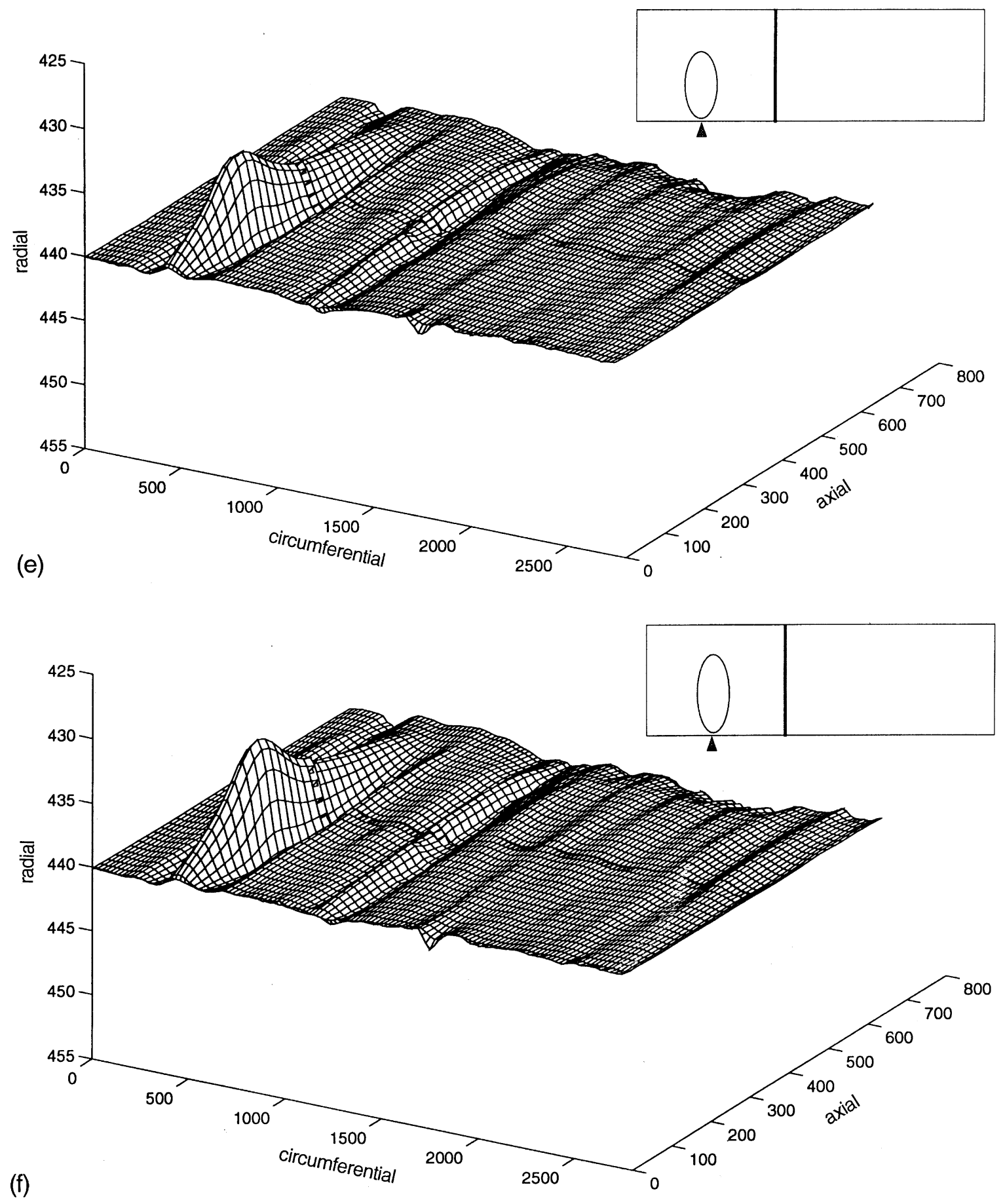

Fig. 9. continued. 
overnight under the load of $350 \mathrm{~N}$; and in the morning it was found to have buckled comprehensively, as in Figs. 1 and 5.

\subsection{Other observations}

A small number of tests was conducted in which no uplift was provided at the base of the shell, but instead a series of weights was applied to the upper disc, equally at opposite ends of a diameter. An elementary calculation showed that the overall out-of-plane bending stiffness of the roof-disc was much less than that of the (practically rigid) shell. Thus the effect of the added weights was to add a component proportional to $\cos 2 \theta$ - where $\theta$ is a circumferential angular coordinate - to the otherwise uniform imposed axial loading. When buckling tests were repeated with larger added weights, the total buckling load was steadily reduced, as if the shell were responding primarily to the largest local loading around the circumference. The results of these tests are shown in Fig. 10. For the sake of simplicity these results have been included in the "imperfect" part of the histogram in Fig. 7.

In another sequence of tests, uplift of different, monotonically increasing amounts was applied for each test at a particular location at the base. In each case, as the axial load on the shell increased, there was an audible "pop" as the first, stable dimple formed. As the uplift increased, the load at which the dimple appeared fell significantly; and yet the buckling load, although some $20 \%$ lower than in the absence of uplift, was insensitive to magnitude of uplift. These observations are plotted in Fig. 11.

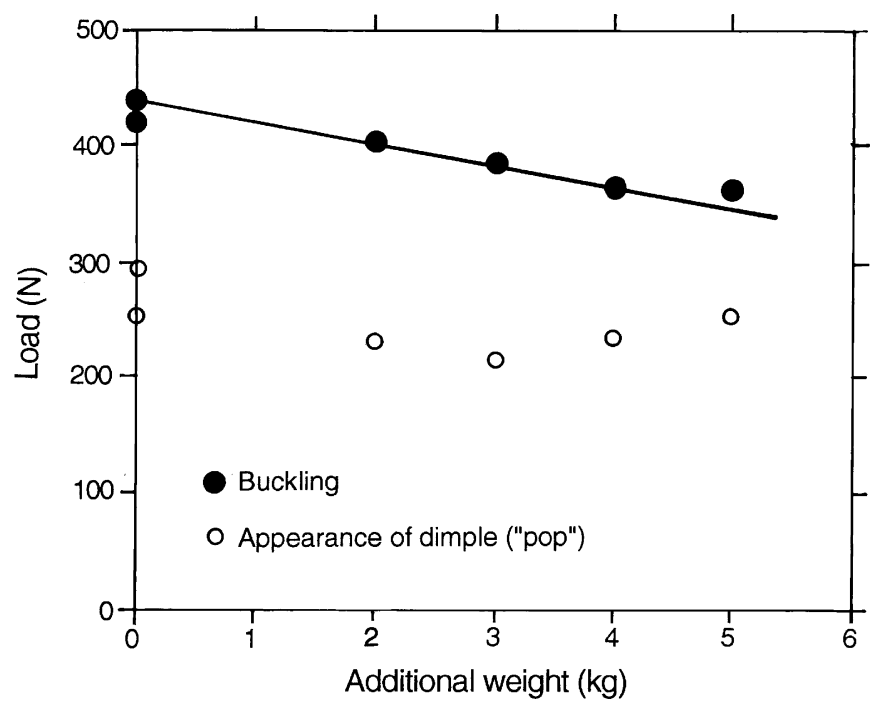

Fig. 10. Plot of buckling load (solid points) against magnitude of equal, diametrically opposed weights placed on the roof. The line corresponds to the hypothesis that the additional weights produce a component of variable axial load intensity around the circumference proportional to $\cos 2 \theta$, and that buckling is determined by the largest local axial load. In these tests no imperfections were deliberately introduced at the base. Also shown (open points) are the corresponding loads at which a dimple first appeared - with an audible "pop". 


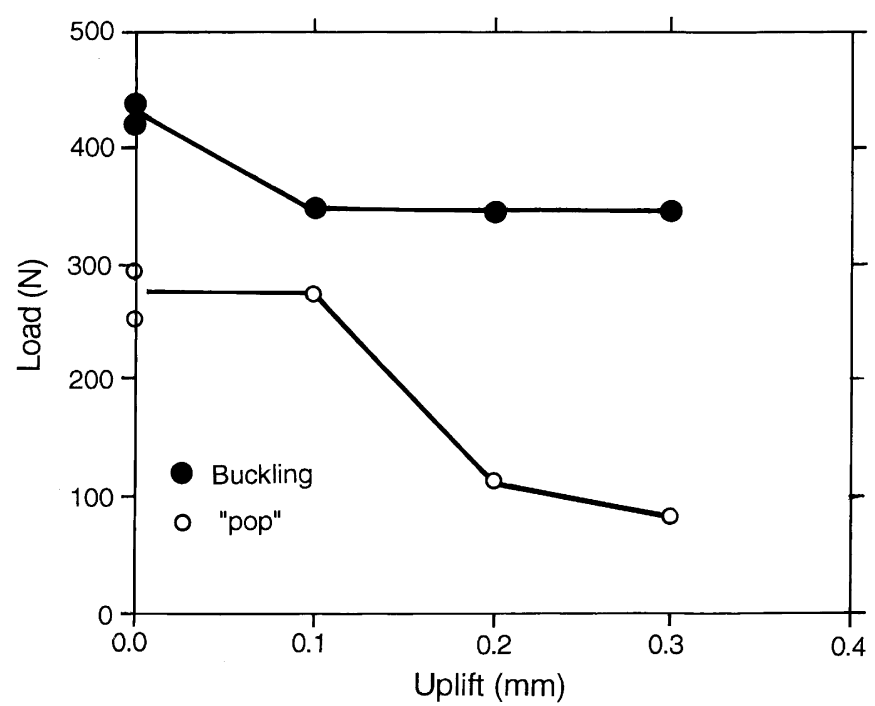

Fig. 11. Plot of buckling load (solid points) against magnitude of uplift at station 30, together with the load (open points) at which the first dimple formed.

\section{Discussion}

How can we begin to explain our various paradoxical observations on the buckling of a cylindrical shell? Our tests revealed practically no "imperfection sensitivity", since tests either with or without deliberate imperfections showed very little "scatter"; and even gross imperfections, in the form of large, stable dimples at zero load, reduced the peak load, on average, by only about $12 \%$.

It seems clear that this unexpected behaviour must be attributed, somehow, to our use of "frictional", rather than "fixed", boundary conditions at the two ends of the shell. As mentioned in Section 2, we introduced this method of fixing the shell to the end-discs primarily for the convenience of being able to dismantle and re-assemble the specimen at will and without difficulty.

Following the exposition of Section 1, a cylindrical shell with both ends held circular and fully fixed in the axial direction (i.e. held plane) is statically indeterminate, whereas the same shell held circular but free to move axially at both its ends is statically determinate. Our own end-fixing arrangements are somewhere in-between these two extremes. Thus, under the frictional clamping action the ends of the shell are held plane for sufficiently small loads. But at higher loads some local slipping may occur, and thus axial displacements are possible without any change in axial force. Hence our particular boundary conditions might be described as "force-limiting" in the axial direction.

An immediate consequence of this is that, if a dimple develops or grows or moves - as in Fig. 9 - there should be practically no consequential changes in the distribution of axial loading around the circumference. This is very different from the case in which the ends of the shell are firmly fixed in the axial direction, where a change in the shape or size of a dimple would be accompanied by 
large local variations in axial stress at the ends of generators passing through the dimple. In other words, the scope for changes in membrane-stress distribution during the creation or enlargement of a dimple is very much reduced when the end-fittings have the "force-limiting" characteristic that is a feature of our type of boundary condition. Indeed, we may speculate that the insensitivity of membrane-stress resultants in the shell to changes of radial displacement - such as the formation of dimples - in the case of frictional end-fittings is related to the overall lack of imperfectionsensitivity of the shell under axial loading.

In relation to the effects of relaxation of the rigidity of end-support, we may mention the work of Librescu and colleagues [15] on the thermal buckling performance of shallow cylindrical panels when the straight or curved edges are restrained tangentially by elastic springs rather than rigidly. Their results are interesting; but there are so many differences in detail between their work and ours that no direct comparisons are possible.

At present, our proposed explanation of the paradoxes revealed by our experimental data is qualitative and hypothetical. However, the hypothesis is testable, and we propose to perform further experiments - probably on smaller specimens - in order to verify and quantify our hypotheses. In this connection we should mention that the experimentally observed self-weight buckling of open-topped thin cylindrical shells - an indisputably "statically determinate" shell structure - and made from silicone rubber shows very little "scatter" in the load/height parameter $[11,12,16]$; which provides some confirmation of our overall hypothesis. We should also mention that recent experiments on buckling of the axially loaded steel shells (drinks cans) into which a local "crease" imperfection had been introduced at mid-height showed that the initial imperfection grew steadily as the load increased, first into a circular and then into an increasingly diamond-shaped stable dimple. Then, when the dimple had reached a particular size - that was practically constant for all tests - the shell buckled in an unstable manner and was covered with dimples [13]. In those experiments the shell was deformed well into the plastic range when the initial damage was inflicted, and it remained in the plastic range throughout the tests. However, it is interesting to note a feature which is common with the present testing, namely that the buckling occurs when an isolated dimple reaches a critical size.

Finally, it has not escaped our notice that the deliberate provision of force-limiting end conditions for practical cylindrical shells such as silos - by means of some ingenious local design feature - might make it possible substantially to increase the load-carrying capacity of some practical thin cylindrical shells.

\section{Acknowledgements}

This work was supported by (UK) SERC, Grant GR/J18811. Mr. R.J. Denston provided invaluable help with the apparatus.

\section{Appendix A. The classical buckling load for the shell}

The classical buckling stress for an axially compressed cylindrical shell of radius $a$ and thickness $t$, made from isotropic elastic material having Young's modulus $E$ and Poisson's ratio $v$ is 
given by

$$
(E t / a) /\left(3\left(1-v^{2}\right)\right)^{1 / 2} \text {. }
$$

Multiplying this by the cross-sectional area of the shell we obtain the classical buckling load as follows:

$$
2 \pi E t^{2} /\left(3\left(1-v^{2}\right)\right)^{1 / 2}
$$

The classical buckling load is thus independent of the radius of the shell.

The melinex sheet was $0.25 \mathrm{~mm}$ thick. Kamyab [8] had determined values of both $E$ and $v$ by performing tensile tests on strips of the material cut from the parent melinex sheet. We accepted his value of 0.3 for $v$, but we decided to make an independent determination of $E$ by tension-loading of strips of material (cut both in the transverse direction on the roll - i.e. the axial direction of the shell - and in the perpendicular direction) and measuring strain by means of a laser extensometer. For strains of up to 0.005 the concensus value of $E$ was $5.0 \mathrm{GN} / \mathrm{m}^{2}$, in good agreement with the value obtained by Holst [14] by performing simple beam-bending tests on strips of the same material.

Using these values we obtain for the classical buckling load of the cylindrical shell $1190 \mathrm{~N}$, or $1200 \mathrm{~N}$ to 2 significant figures.

\section{References}

[1] von Kármán T, Dunn LG, Tsien H-S. The influence of curvature on the buckling characteristics of structures. Journal of the Aeronautical Sciences 1940;7:276-89.

[2] Koiter WT. The stability of elastic equilibrium. Dissertation for the degree of Docor in the Technical Sciences at the Technische Hooge School, Delft, 1945. Amsterdam: H.J. Paris (in Dutch). English translation by E. Riks (1970): Technical report AFFDL-TR-70-25, Air Force flight dynamics laboratory, Air Force Systems Command, WrightPatterson Air Force Base, Ohio, USA.

[3] Arbocz J. The effect of initial imperfections on shell stability. In: Fung YC, Sechler EE, editors. Thin shell structures. Englewood Cliffs, NJ: Prentice-Hall Inc., 1974. p. 205-45.

[4] Abramovich H, Singer J, Weller T. The influence of initial imperfections on the buckling of stiffened cylindrical shells under combined loading. In: Jullian JF, editor. Buckling of shell structures on land, in the sea, and in the air. London: Elsevier Applied Science, 1991. p. 1-10.

[5] Heyman J. Equilibrium of shell structures. Oxford: Clarendon Press, 1977.

[6] Pellegrino S, Calladine CR. Matrix analysis of statically and kinematically indeterminate frameworks. International Journal of Solids Structures 1986;22:409-28.

[7] Calladine CR. Theory of shell structures. Cambridge: Cambridge University Press, 1983.

[8] Kamyab H, Palmer SC. Experimental investigation of the displacements in oil storage tanks caused by differential settlement. In: Allison IM, Ruiz C, editors. Applied solid mechanics, vol. 3. London: Elsevier Applied Science, 1989. p. 336-50.

[9] Brush DO, Almroth BO. Buckling of bars, plates and shells. New York: McGraw-Hill Book Co., 1975.

[10] Koiter WT. The effect of axisymmetric imperfections on the buckling of cylindrical shells under axial compression. Proc. Koninklijke Nederlandse Akademie van Wetenschappen 1963;66(b):265-79.

[11] Calladine CR, Barber JN. Simple experiments on self-weight buckling of open cylindrical shells. Journal of Applied Mechanics 1970;37:1150-1.

[12] Mandal P. PhD Dissertation, University of Cambridge, 1997.

[13] Hambly ET, Calladine CR. Buckling experiments on damaged cylindrical shells. International Journal of Solids Structures 1996;33:3539-48. 
[14] Holst JMFG. PhD Dissertation, University of Cambridge, 1996.

[15] Librescu L, Liu W, Nemeth MP, Starnes JH. Thermomechanical postbuckling of geometrically imperfect flat and curved panels taking into account tangential edge constraints. Journal of Thermal Stresses 1995;18:465-82.

[16] Mandal P, Calladine CR. Buckling on thin cylindrical shells under axial compression. International Journal of Solids and Structures 1999, in press. 